Z Gerontol Geriat 2015 • 48:600

DOI 10.1007/s00391-015-0957-1

Online publiziert: 18. September 2015

(c) Springer-Verlag Berlin Heidelberg 2015
Hans Jürgen Heppner ${ }^{1}$ Markus Gosch ${ }^{2}$

${ }^{1}$ Geriatrische Klinik und Tagesklinik, Helios Klinik Schwelm, Universität Witten/Herdecke,

Schwelm, Deutschland

${ }^{2}$ Universitätsklinik für Geriatrie, Paracelsus Medizinische Privatuniversität, Nürnberg, Deutschland

\title{
Erratum zu: Alter und Infektionen
}

\section{Seit jeher zwei auf das Engste verknüpfte Themen}

\section{Erratum zu:}

Z Gerontol Geriat 2015

DOI 10.1007/s00391-015-0944-6

In der zunächst veröffentlichten OnlineVersion des Beitrags wurde bei der Bearbeitung im Verlag der Name des Autors Hans Jürgen Heppner technisch falsch gekennzeichnet. Der Vorname muss Hans Jürgen lauten und der Nachname Heppner.

Die Redaktion bittet, den Fehler zu entschuldigen und um Beachtung der korrekten Version.

\section{Korrespondenzadresse}

\section{Prof. H.J. Heppner}

Geriatrische Klinik und Tagesklinik Helios Klinik Schwelm, Universität Witten/ Herdecke

Dr.-Moeller-Str. 15, 58332 Schwelm

Hans.Heppner@uni-wh.de

Prof. Dr. M. Gosch

Universitätsklinik für Geriatrie,

Paracelsus Medizinische Privatuniversität

Klinikum Nürnberg

Prof.-Ernst-Nathan-Str. 1, 90419 Nürnberg

markus.gosch@klinikum-nuernberg.de 